Supporting Information

\title{
Thermoplastic "all-cellulose" composites with covalently attached carbonized cellulose
}

Lotta H. Gustavsson', Karin H. Adolfsson, Minna Hakkarainen*

Department of Fibre and Polymer Technology, KTH Royal Institute of Technology,

Teknikringen 58, 10044 Stockholm, Sweden

Content:

1. Characterization of ionic liquid AmimCl

2. Dissolution of cellulose

3. Characterization of oxidized carbonized cellulose (OCC)

4. Characterization of composite and reference products

5. Analysis of remaining acetic anhydride after acetylation of cellulose

6. XPS results for OCC, OCC-Cl, CAL-OCC 2 and CAL $+2 \mathrm{wt} \%$ OCC 
1. Synthesis of ionic liquid $\mathrm{AmimCl}^{1}$

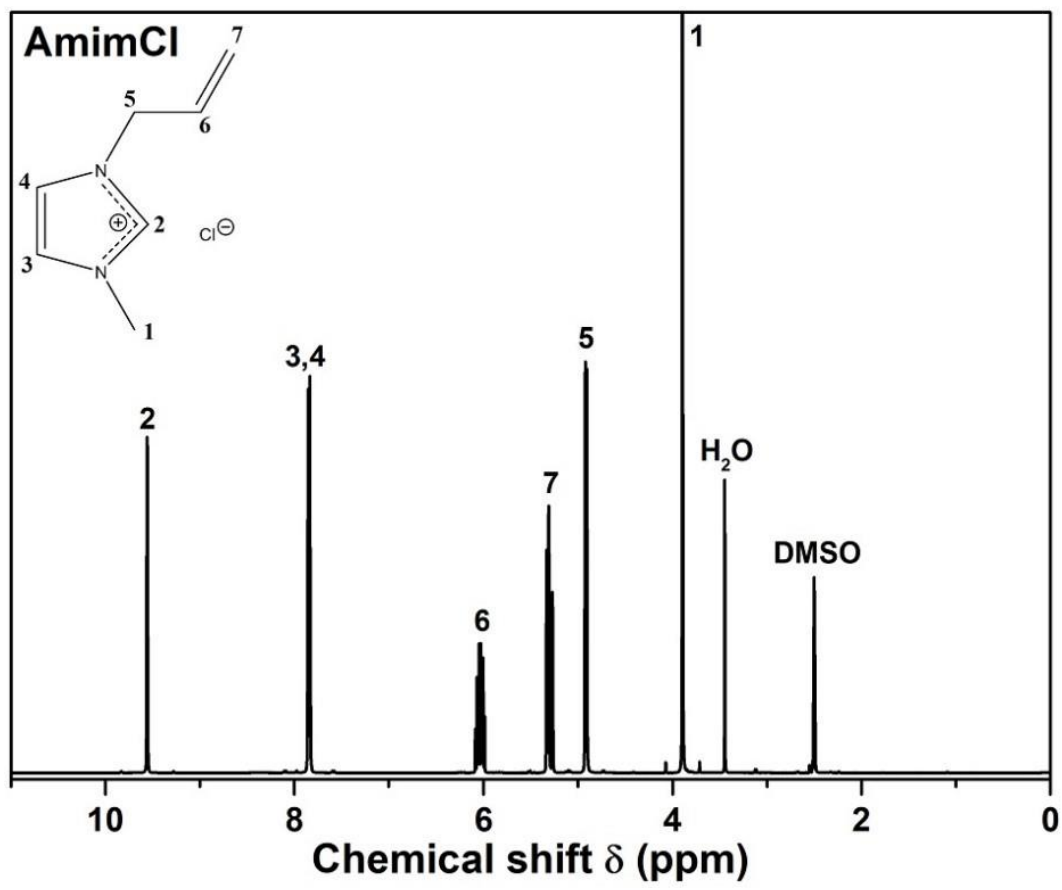

Figure S1. ${ }^{1} \mathrm{H}$ NMR of AmimCl.

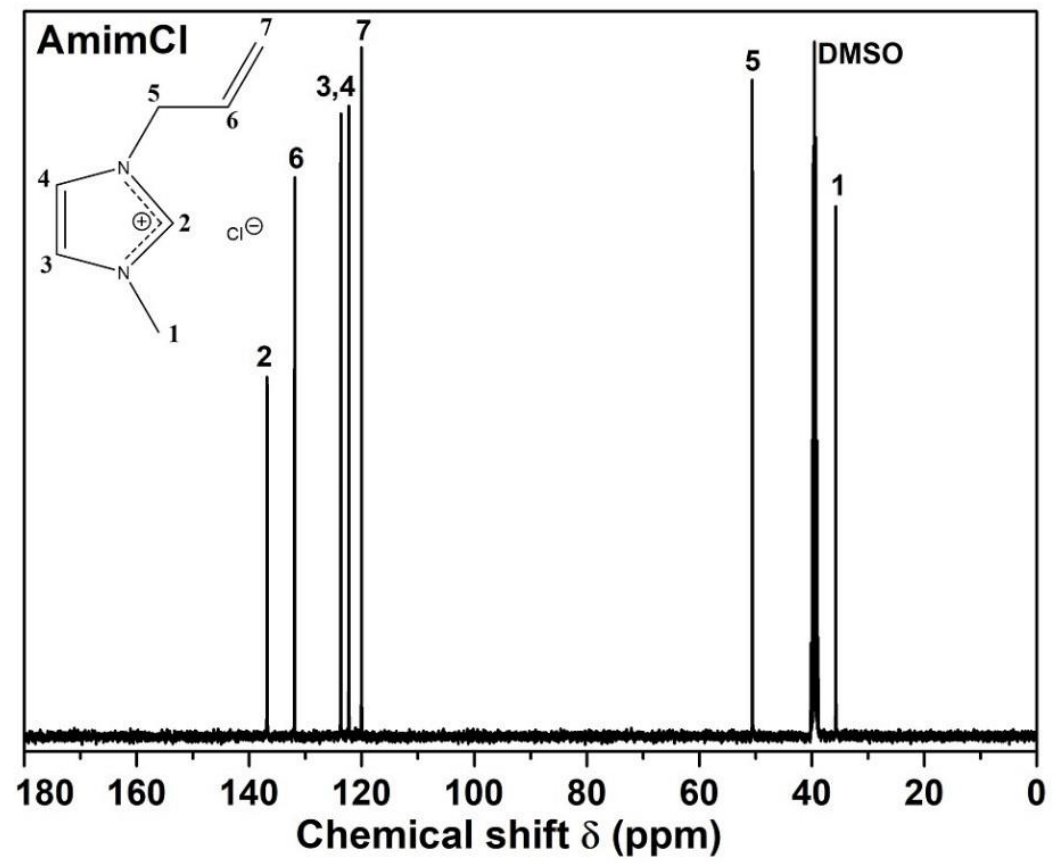

Figure S2. ${ }^{13} \mathrm{C}$ NMR of $\mathrm{AmimCl}$. 


\section{Dissolution of cellulose}
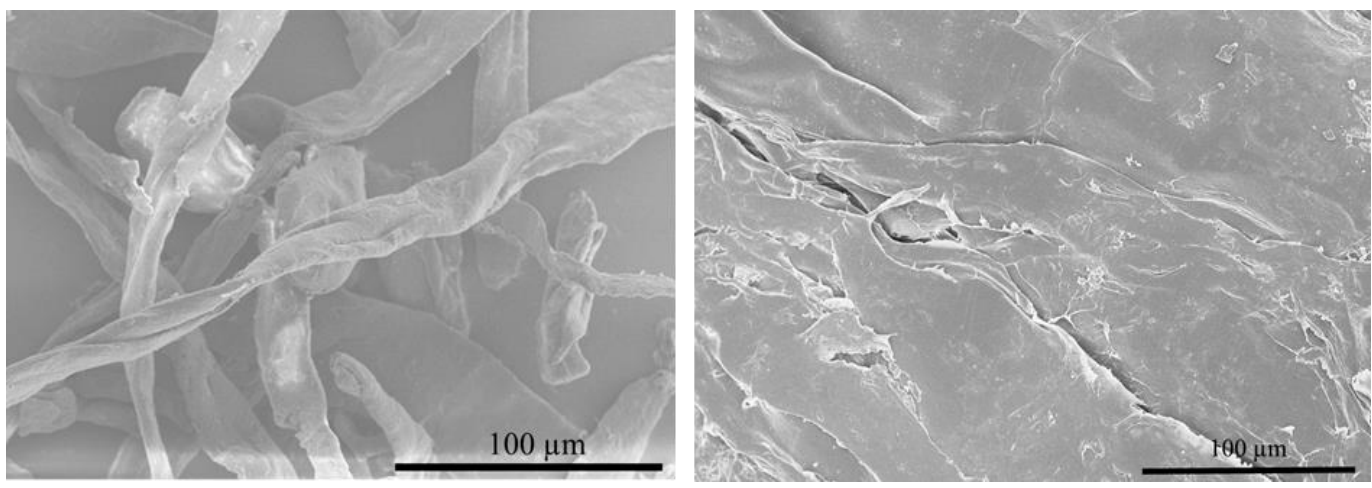

Figure S3. SEM images of cellulose before (on the left) and after (on the right) the dissolution. The fibrous structure is seen on the pristine cellulose, whereas regenerated cellulose has lost its fibre structures.

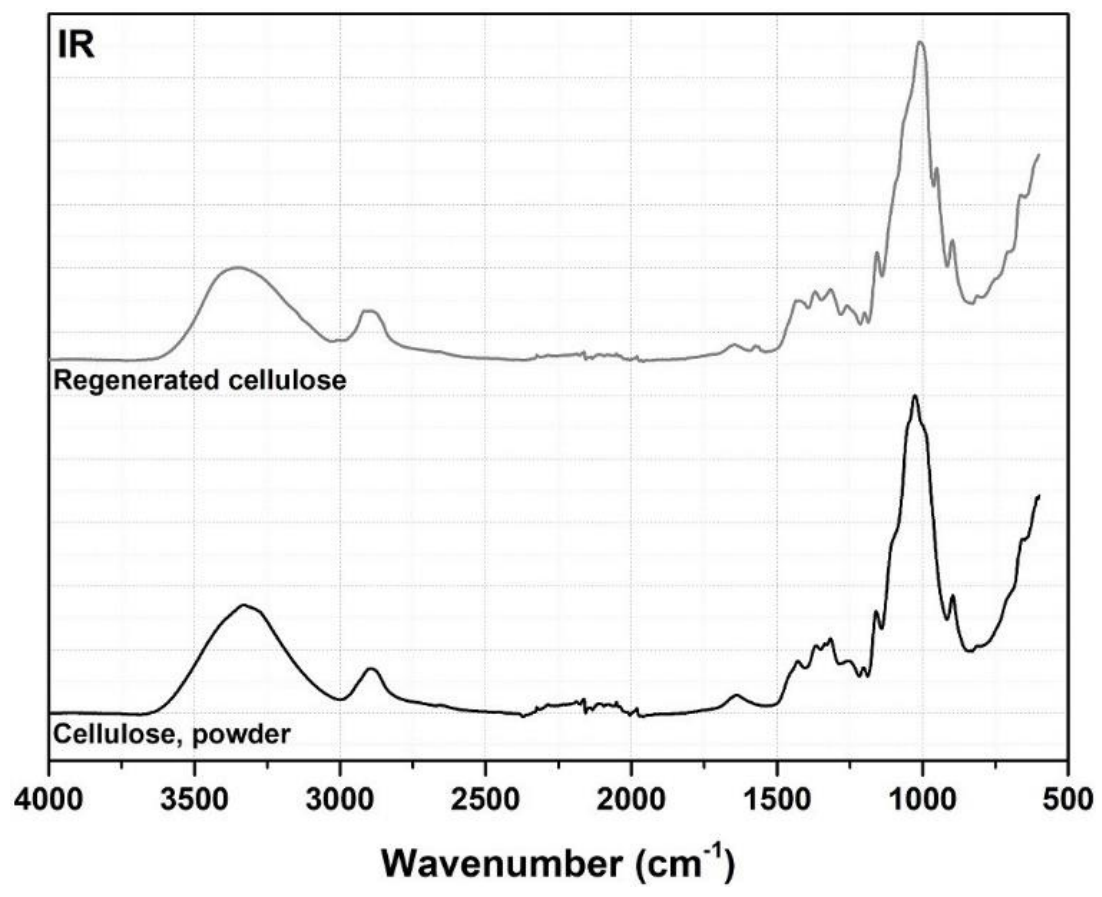

Figure S4. FTIR spectra of pristine $\alpha$-cellulose and regenerated cellulose. 
3. Characterization of oxidized carbonized cellulose (OCC)

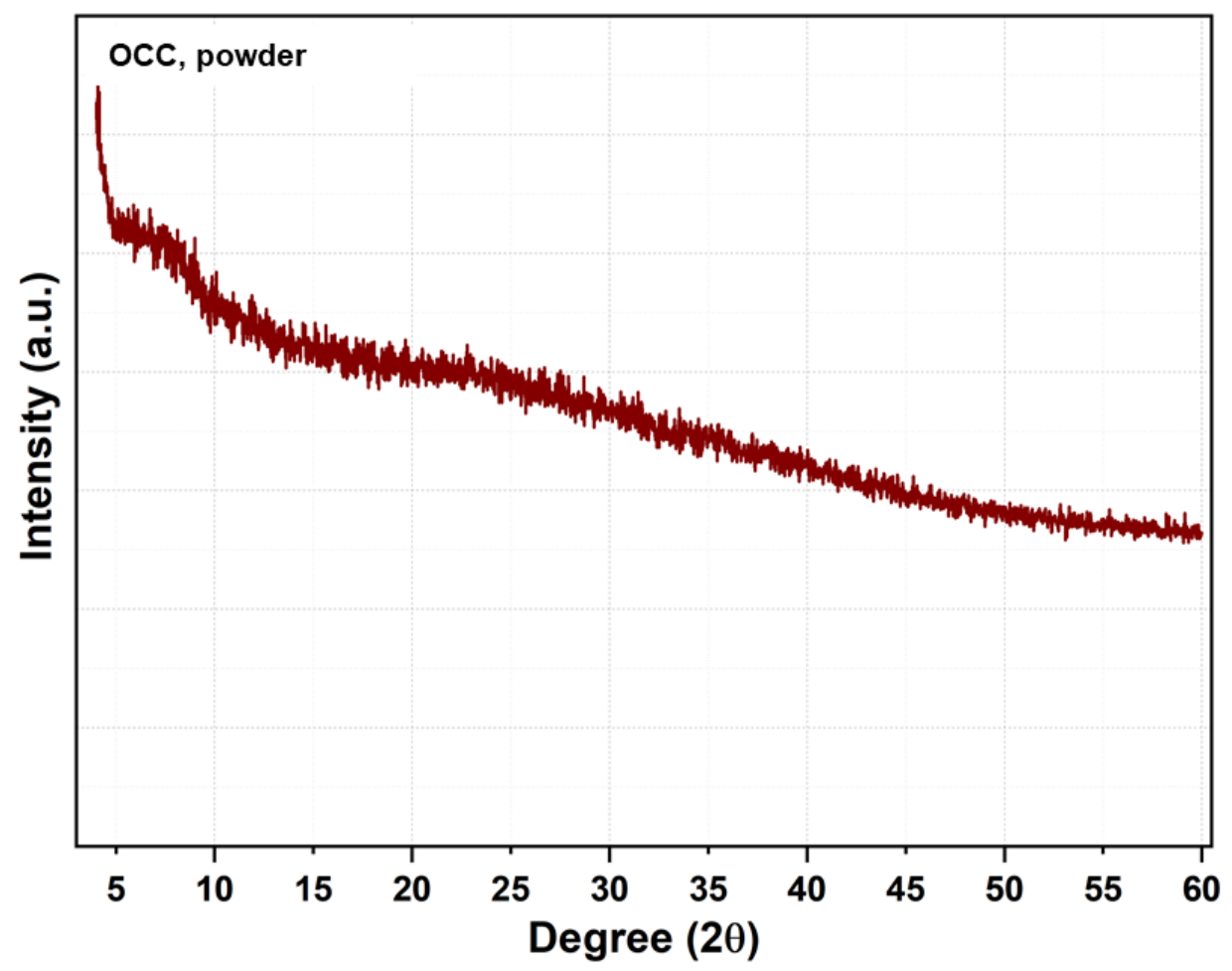

Figure S5. WAXD of OCC.

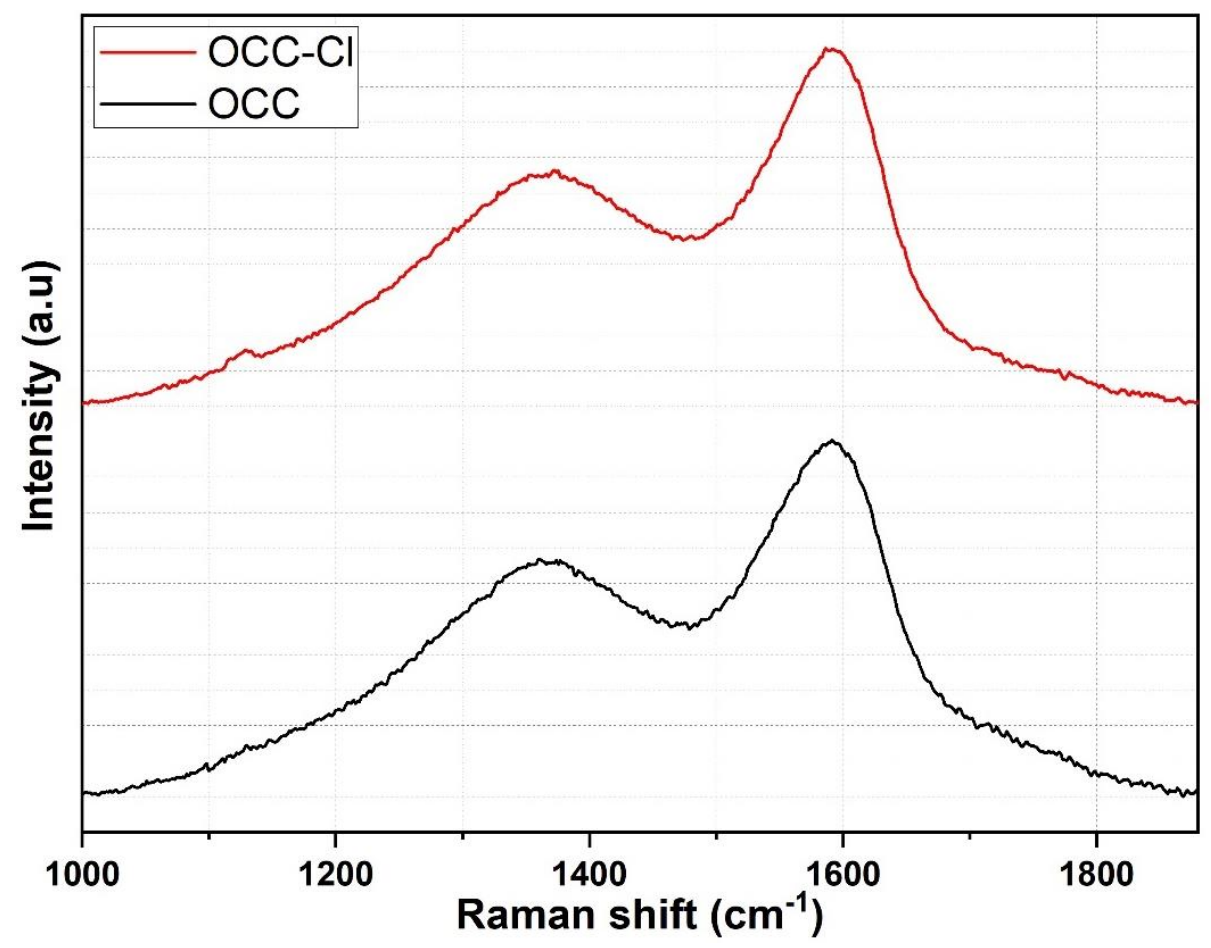

Figure S6. Raman spectra of OCC and OCC-Cl. 
4. Characterization of composite and reference products ${ }^{2,3}$

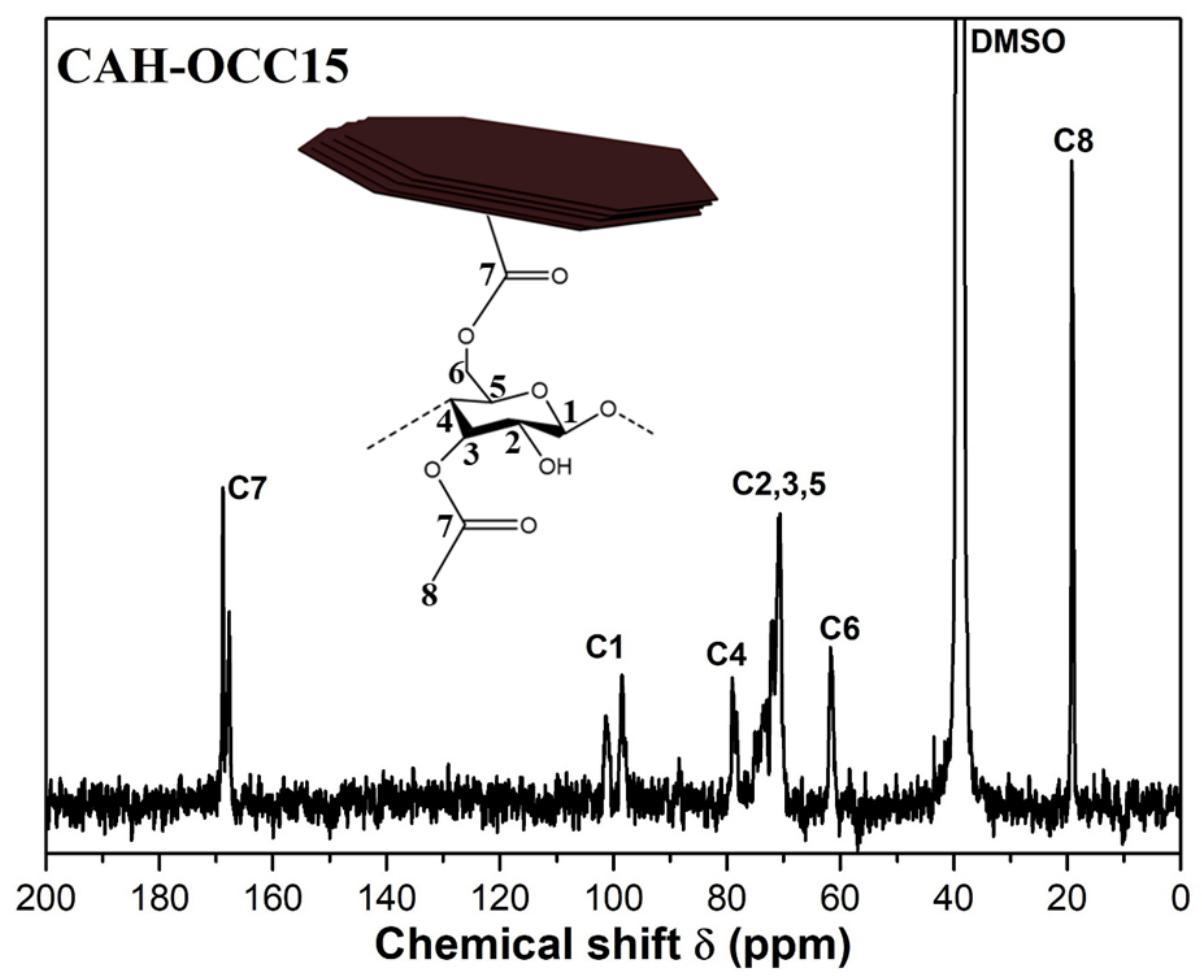

Figure S7. ${ }^{13} \mathrm{C}$ NMR spectrum of CAH-OCC15.

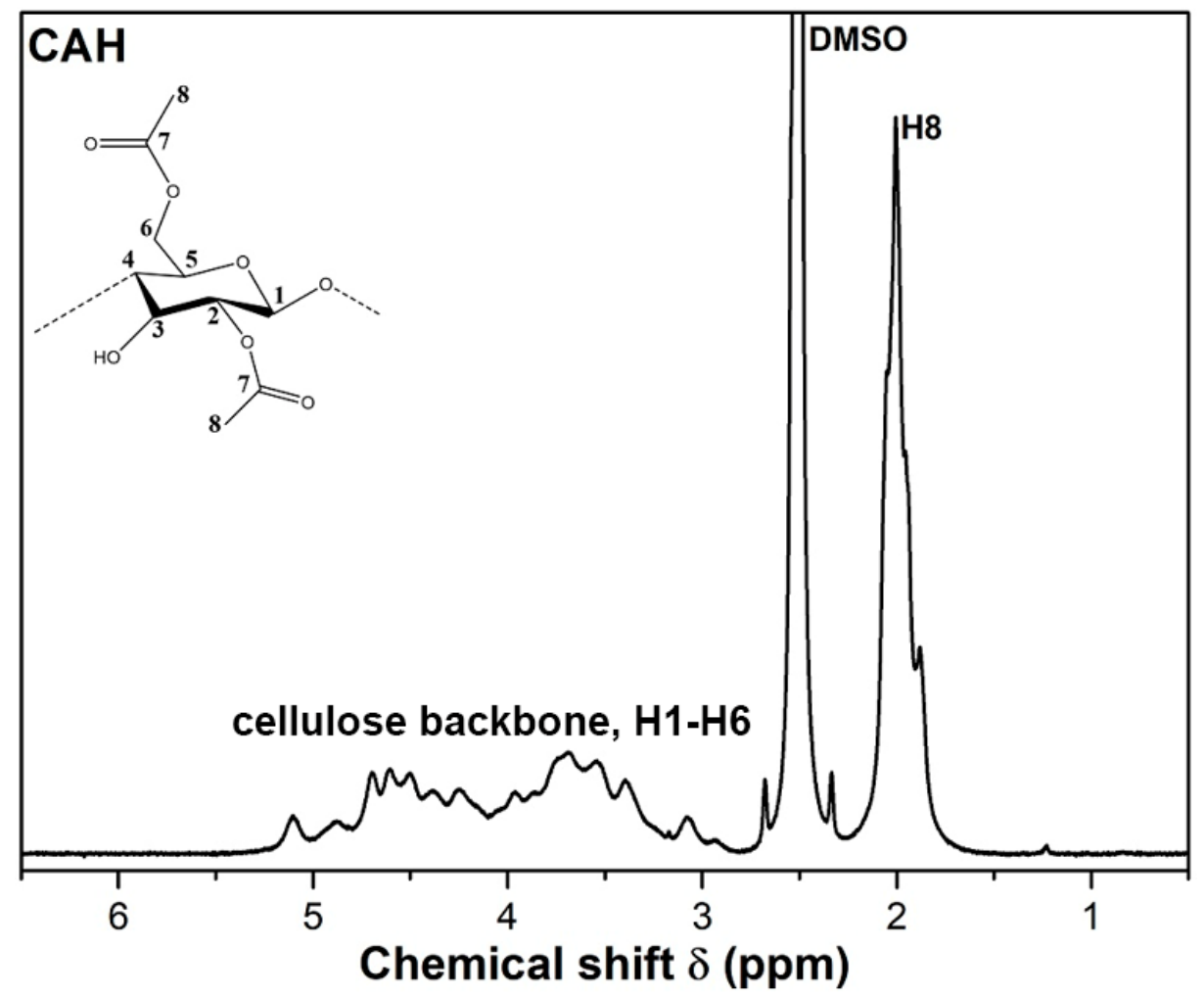

Figure S8. ${ }^{1} \mathrm{H}$ NMR of the reference compound CAH. 


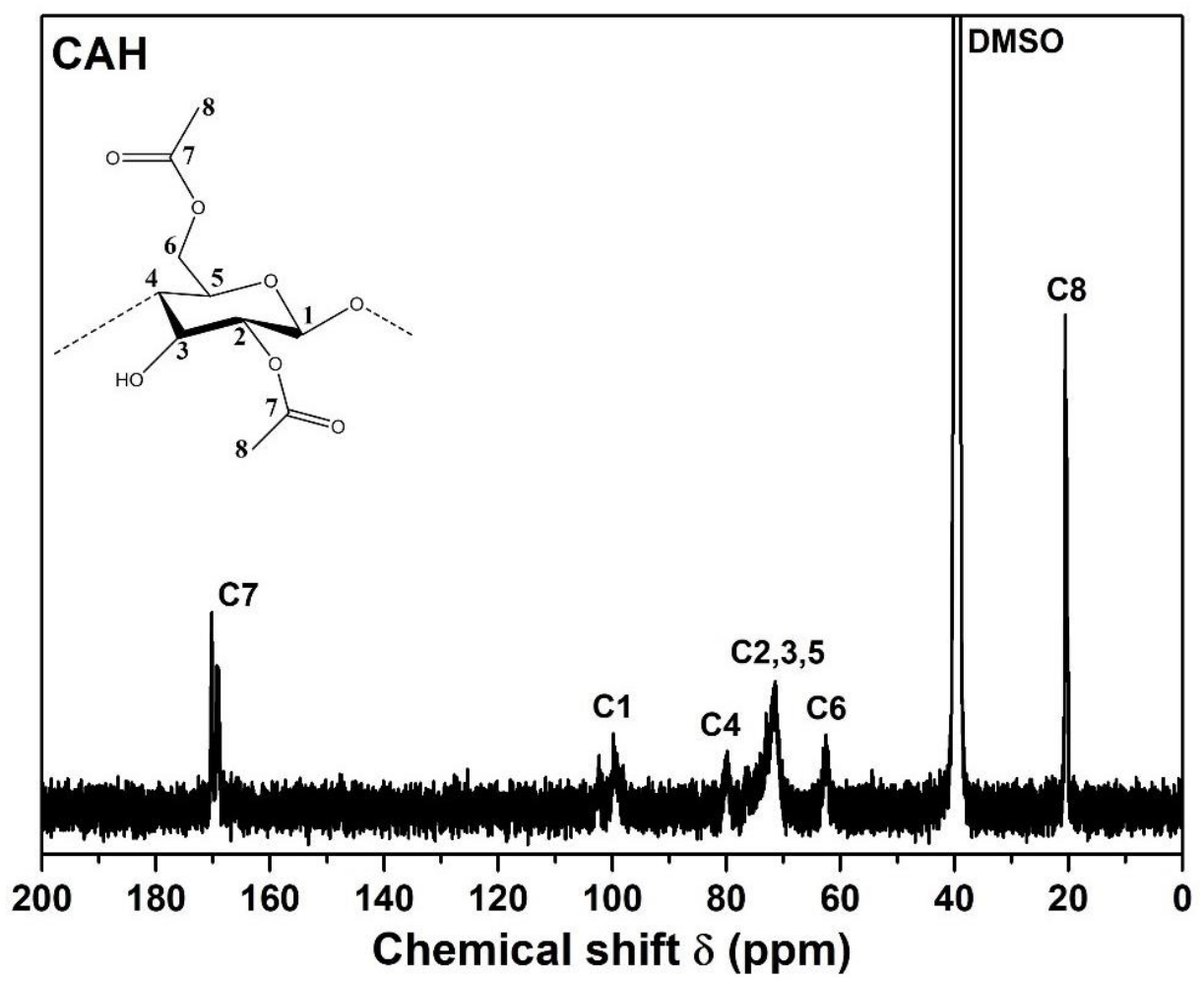

Figure S9. ${ }^{13} \mathrm{C}$ NMR of the reference compound CAH.

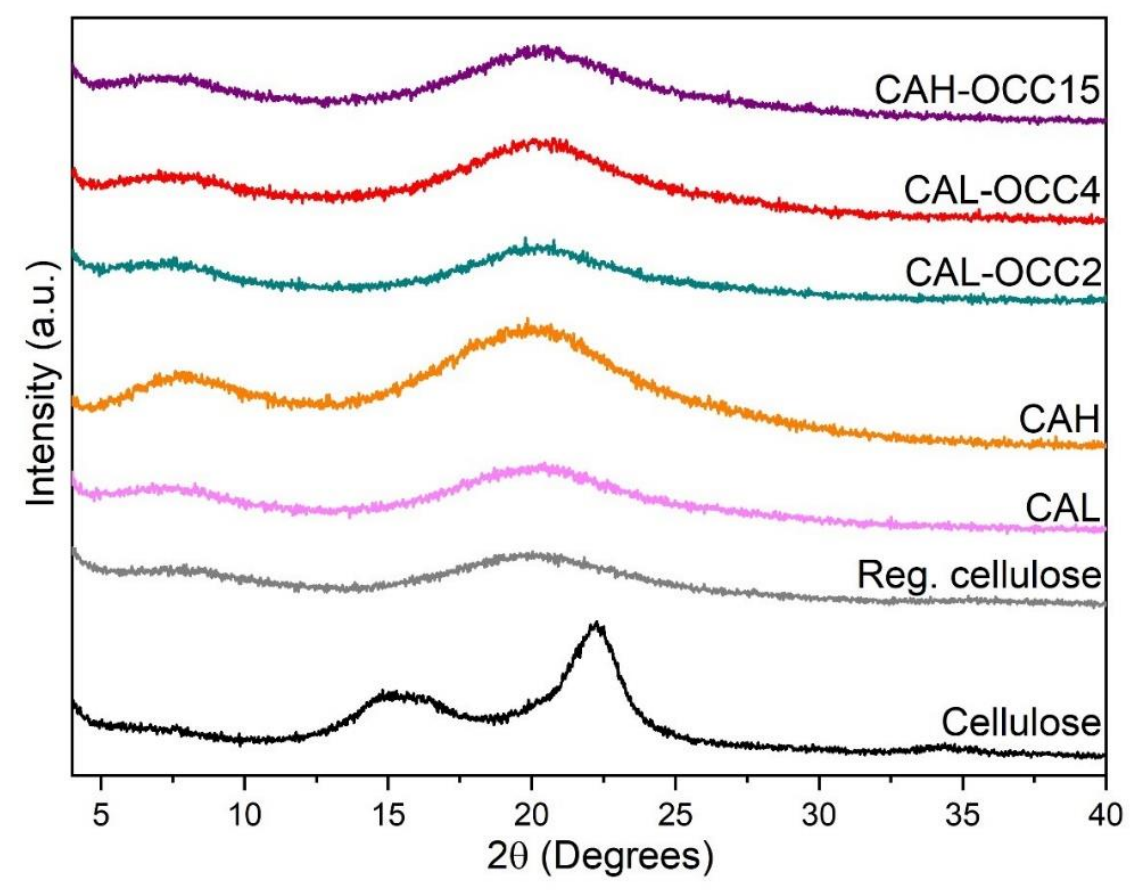

Figure S10. WAXD spectra of the starting $\alpha$-cellulose, regenerated cellulose from $\mathrm{AmimCl}$ and the synthesized composites and references. 


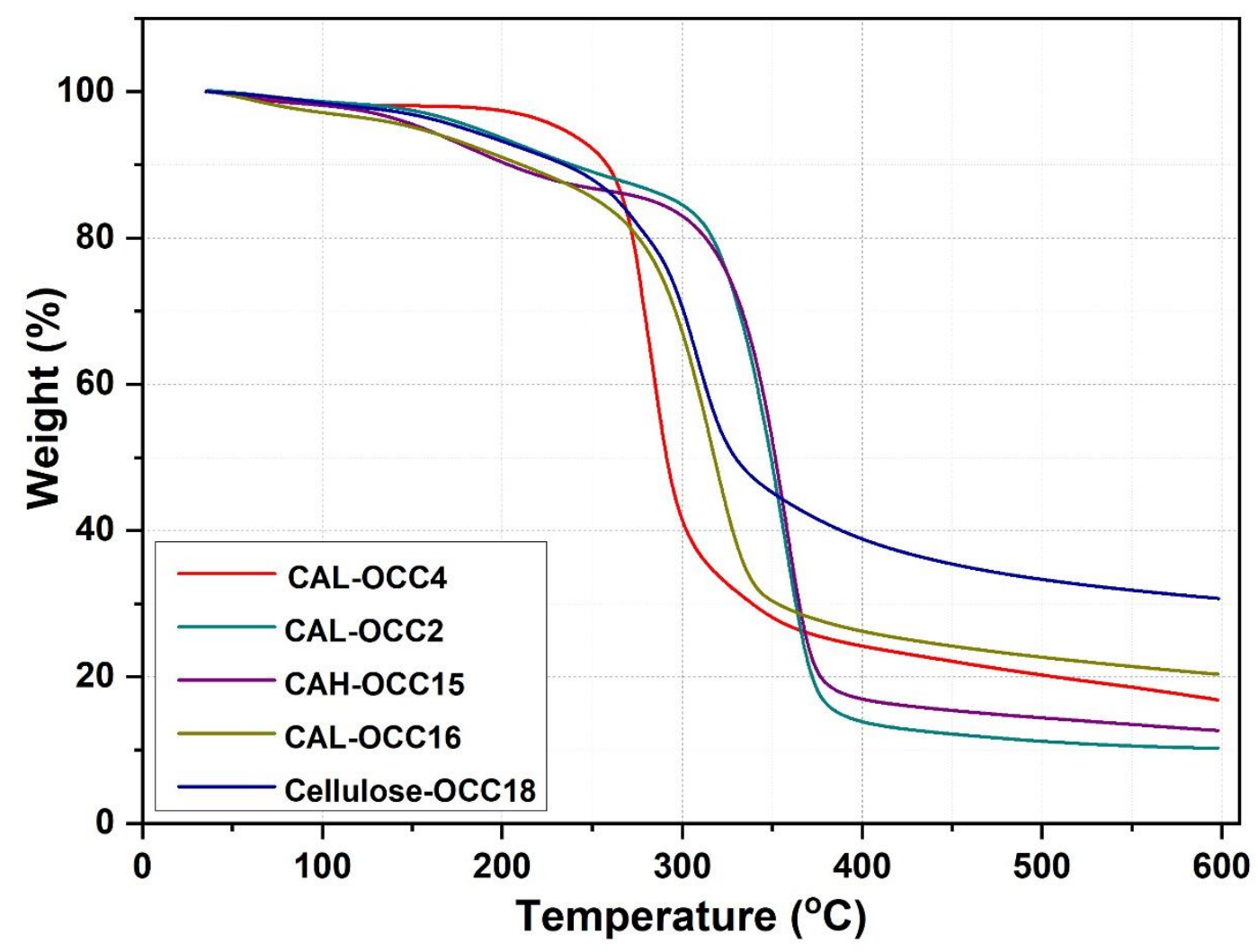

Figure S11. TGA of CA-OCC composites and cellulose-OCC composite.

Table S1. Results from tensile testing of CAL-OCC2.

\begin{tabular}{|cccc|} 
Sample & $\begin{array}{c}\text { Stress at } \\
\text { break } \\
\text { (MPa) }\end{array}$ & $\begin{array}{c}\text { Extension } \\
\text { at break } \\
\mathbf{( \% )}\end{array}$ & $\begin{array}{c}\text { Young's } \\
\text { modulus } \\
\text { (GPa) }\end{array}$ \\
\hline $\mathbf{1}$ & 78.39 & 14.03 & 1.69 \\
\hline $\mathbf{2}$ & 72.58 & 9.79 & 1.50 \\
\hline $\mathbf{3}$ & 72.89 & 10.29 & 1.62 \\
\hline $\mathbf{4}$ & 65.50 & 10.62 & 1.39 \\
\hline $\mathbf{5}$ & 67.50 & 9.46 & 1.47 \\
\hline $\mathbf{6}$ & 71.62 & 11.79 & 1.52 \\
\hline Average & $\mathbf{7 1 . 4 1} \pm \mathbf{6 . 9 8}$ & $\mathbf{1 1 . 0 0} \pm \mathbf{3 . 0 9}$ & $\mathbf{1 . 5 3} \pm \mathbf{0 . 1 5}$ \\
\hline
\end{tabular}


5. Analysis of remaining acetic anhydride after acetylation of cellulose

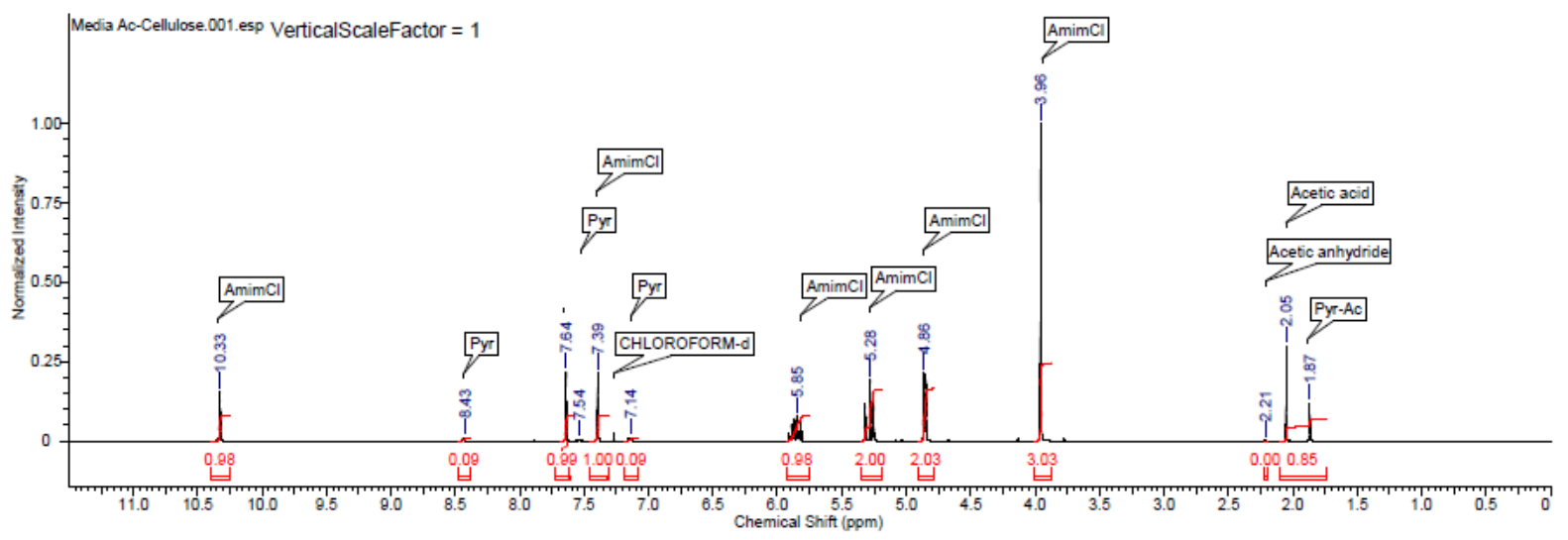

Figure S12. ${ }^{1} \mathrm{H}$ NMR of reaction mixture after synthesis of neat $\mathrm{CA}$ of DS=1.4.

The analysis of the spectrum shows that acetic anhydride is nearly completely consumed after $2 \mathrm{~h}$ of acetylation, producing acetic acid and pyridinium acetate. The peak of remaining acetic anhydride has integral $\mathrm{I}=0.00$, which suggests a solution of less than $1 \mathrm{~mol}-\%$ acetic anhydride in AmimCl. This corresponds to the consumption of $\sim 95 \%$ of the original acetic anhydride. ${ }^{4}$ 
6. XPS results for OCC, OCC-CI, CAL-OCC2 and CAL + 2 wt \% OCC

Table S2. At $\%$ for OCC, OCC-Cl, CAL-OCC 2 and CAL $+2 \mathrm{wt} \%$ OCC.

\begin{tabular}{|c|c|c|c|c|c|c|c|}
\hline at $\%$ & C 1s & O 1s & $\mathbf{N} 1 \mathrm{~s}$ & Si 2p & Cl 2p & Ca $2 p$ & S 2p \\
\hline OCC & 72.1 & 22.8 & 3.1 & 1.8 & & & 0.3 \\
\hline OCC-Cl & 64.0 & 25.2 & 2.9 & 4.7 & 2.5 & & 0.6 \\
\hline CAL-OCC2 & 68.1 & 23.0 & 0.4 & 8.2 & & 0.3 & \\
\hline CAL $+2 w t \%$ OCC & 64.0 & 33.0 & 0.5 & 2.5 & & & \\
\hline
\end{tabular}

Table S3. Binding energies (BE), full-width at half maximum (FWHM) and $\mathrm{O} / \mathrm{C}$ ratios from narrow scans $\mathrm{C} 1 \mathrm{~s}$ for OCC, OCC-Cl, CAL-OCC2 and CAL $+2 \mathrm{wt} \%$ OCC.

Sample BE (eV) FWHM (eV) Suggested $\quad$ O/C assignments ratio

\begin{tabular}{|c|c|c|c|c|}
\hline \multirow[t]{4}{*}{ OCC } & 285.2 & 1.5 & $\mathrm{C}-\mathrm{C} / \mathrm{C}=\mathrm{C}$ & 0.32 \\
\hline & 286.7 & 1.7 & $\mathrm{C}-\mathrm{O}$ & \\
\hline & 288.1 & 1.7 & $\mathrm{C}-\mathrm{O}-\mathrm{C} / \mathrm{C}=\mathrm{O}$ & \\
\hline & 289.3 & 1.7 & $\mathrm{O}=\mathrm{C}-\mathrm{O}$ & \\
\hline \multirow[t]{4}{*}{ OCC-Cl } & 285.2 & 2.0 & $\mathrm{C}-\mathrm{C} / \mathrm{C}=\mathrm{C}$ & 0.39 \\
\hline & 286.7 & 2.1 & $\mathrm{C}-\mathrm{O}$ & \\
\hline & 288.1 & 2.1 & $\mathrm{C}-\mathrm{O}-\mathrm{C} / \mathrm{C}=\mathrm{O}$ & \\
\hline & 289.3 & 2.1 & $\mathrm{O}=\mathrm{C}-\mathrm{O}$ & \\
\hline \multirow{4}{*}{ CAL-OCC2 } & 284.8 & 1.2 & $\mathrm{C}-\mathrm{C} / \mathrm{C}=\mathrm{C}$ & 0.34 \\
\hline & 286.7 & 1.3 & $\mathrm{C}-\mathrm{O}$ & \\
\hline & 288.1 & 1.3 & $\mathrm{C}-\mathrm{O}-\mathrm{C} / \mathrm{C}=\mathrm{O}$ & \\
\hline & 289.3 & 1.3 & $\mathrm{O}=\mathrm{C}-\mathrm{O}$ & \\
\hline \multirow{4}{*}{$\mathrm{CAL}+2 \mathrm{wt} \% \mathrm{OCC}$} & 284.9 & 1.6 & $\mathrm{C}-\mathrm{C} / \mathrm{C}=\mathrm{C}$ & 0.52 \\
\hline & 286.7 & 1.2 & $\mathrm{C}-\mathrm{O}$ & \\
\hline & 288.1 & 1.2 & $\mathrm{C}-\mathrm{O}-\mathrm{C} / \mathrm{C}=\mathrm{O}$ & \\
\hline & 289.3 & 1.2 & $\mathrm{O}=\mathrm{C}-\mathrm{O}$ & \\
\hline
\end{tabular}



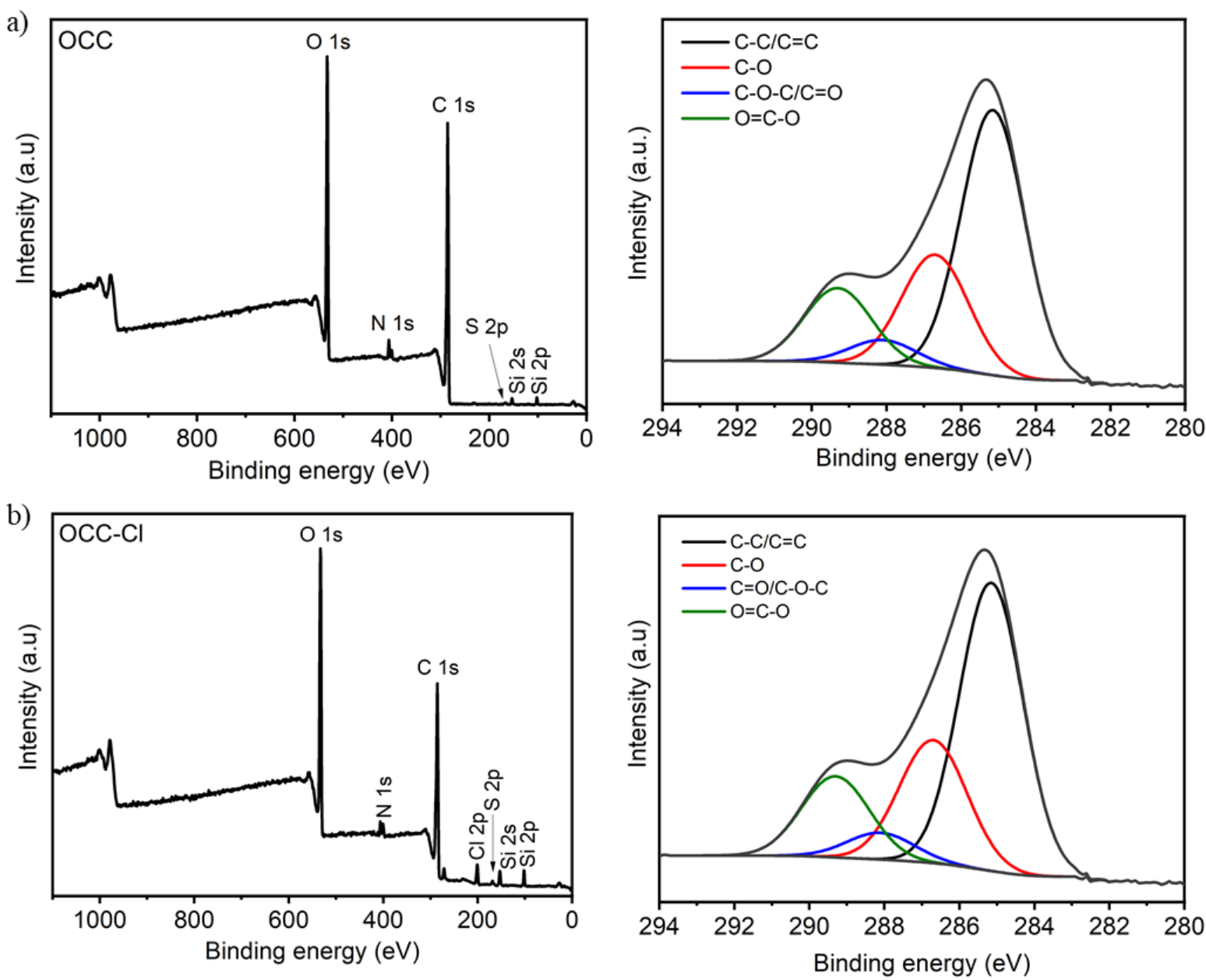

Figure S13. XPS survey spectra (left) and narrow scans (right) for (a) OCC and (b) OCC-Cl.

\section{References}

1. Zhang, H.; Wu, J.; He, J., 1-Allyl-3-methylimidazolium Chloride Room Temperature Ionic Liquid: A New and Powerful Nonderivatizing Solvent for Cellulose. Macromolecules 2005, $38(20), 8272-8277$.

$2 . \quad$ Chen, Z.; Zhang, J.; Xiao, P.; Tian, W.; Zhang, J., Novel Thermoplastic Cellulose Esters Containing Bulky Moieties and Soft Segments. ACS Sustainable Chem. Eng. 2018, 6, 49314939.

$3 . \quad$ Barthel, S.; Heinze, T., Acylation and Carbanilation of Cellulose in Ionic Liquids. Green Chem. 2005, 8, 301-306.

4. Nowrouzi, N.; Farahi, S.; Irajzadeh, M., 4-(N,N-Dimethylamino)pyridiniumacetate as a recyclable catalyst for the synthesis of 5 -substituted-1H-tetrazoles. Tetrahedron Lett. 2015, 56 (5), 739-742. 